\title{
Temperature and strain rate dependent mechanical response of METCO 601 aluminium-polyester abradable seal coating
}

\author{
Antonio Pellegrino, ${ }^{1,}$, Maria Jesus Perez-Martin ${ }^{1}$, Kalin Dragnevski ${ }^{1}$, Giuseppe Zumpano ${ }^{2}$, and Nik Petrinic ${ }^{1}$ \\ ${ }^{1}$ University of Oxford, Department of Engineering Science, Parks Road, Oxford OX1 1PJ, UK \\ ${ }^{2}$ Rolls-Royce plc, P.O. box 31, Derby DE24 8BJ, UK
}

\begin{abstract}
Abradable coatings are utilised as sacrificial materials in low-pressure compressor casings, and in intermediate and high pressure compressors and seals. The adoption of abradable coatings allows for the clearance between compressor blades and casing to be minimised, increasing the overall efficiency of the engine. Quasi-static and dynamic experiments at different temperatures are conducted to characterise the mechanical response of a thermally-sprayed abradable seal coating. The material is composed of an aluminium continuum matrix and fairly well-dispersed polyester particles. Stress versus strain histories are measured in uniaxial tension and compression at strain rates ranging from $10^{-3}$ to $10^{2} \mathrm{~s}^{-1}$, via non-standard experimental techniques. The material displays sensitivity to the strain rate and to the imposed temperature. The mechanical behaviour is brittle in tension while it exhibits higher strains to failure in compression. The material is characterised by a pronounced tension/compression asymmetry.
\end{abstract}

\section{Introduction}

Coatings are used in all engineering design scenarios when the desired surface properties are different from the desired structural material properties. Gas turbine coatings are employed in aerospace propulsion in various sections of jet engines subjected to different temperature, pressure and loading conditions for several purposes [1]. Their primary applications include fretting wear protection, thermal barrier, corrosion, oxidation and wear resistance enhancement. Fretting wear resistant coatings are utilised at the contact area between compressors blade roots and disc. Thermal barrier coatings are utilised to protect combustion chamber, turbine blades and vanes and the exit nozzle. Corrosion and oxidation resistant coatings preserve blades and vanes from oxidation. Erosion and wear resistant coatings are applied to compression blades and vanes and high pressure compressors drum and seal runners. Abradable coatings are utilised as sacrificial materials in low pressure compressor casings, and in intermediate and high pressure compressors and seals.

The adoption of abradable coatings allows for the clearance between compressor blades and casing to be minimised, increasing the gas path efficiency of gas turbines [1-2]. For this reason, they are used both for aerospace and power generation applications. During jet engine operation the concurrent effects of centrifugal forces generated by the rotational speed, thermal expansion, minimal shaft misalignments, slight ovalisation of the casing and tight geometrical tolerances can lead to the contact between blades and casing. The application of abradable coatings to the containment avoids this contact to occur between metals, with detrimental consequences on the operational life of the engine [3-6]. These coatings act as sacrificial materials and their mechanical properties are characterised by an optimal compromise between good abradability and adequate resistance to the erosion of ingested particles and high speed gas flow.

METCO 601 is a plasma sprayed coating composed of blend of silicon-aluminium and polyester powders [7]. Its microstructure comprises an aluminium metal phase, fairly uniformly dispersed particles of polyester and voids. The aluminium confers the coating good mechanical (bond strength, inter-particle strength) and thermal properties. The polyester confers the material good abradability and reduces its coefficient of friction. This type of coating finds application in jet engines compressors made of titanium, operating at temperatures up to $325^{\circ} \mathrm{C}$, being the high temperature limit posed by the melting temperature of the polymer.

Previous studies have investigated the mechanical behaviour and the wear performance of METCO 601 . The elastic modulus of METCO 601 and of a number of other Sulzer Metco abradable coatings were obtained using a cantilever beam bending setup on specimens sprayed on a steel substrate [4]. The ultimate tensile strength was inferred via tensile tests in [6], where plasma sprayed specimens on grit blasted mild steel were used. Information relevant to the erosion and wear behaviour were obtained in [3, 5], where sliding wear tests were conducted on a MM-200 wear testing machine and a bespoke developed erosion test machine was developed to assess the effects of erosion time, impact angle and velocity of abrasive particles on the erosion

Corresponding author: antonio.pellegrino@eng.ox.ac.uk 
rate of several abradable seal coatings. The variation of the energy dissipated per unit of abraded volume of coating during scratch tests was evaluated in [6].

However, despite the extensive research on abradable coatings, no studies investigated the dynamic behaviour of METCO 601 and the dependence of its mechanical response upon temperature. This information is needed in order to predict accurately its mechanical behaviour in operating conditions. In order to overcome this gap, a series of experiments on METCO 601 specimens separated from any substrate were conducted at different temperatures and strain rates using a universal testing machine and a long Hopkinson bar setup provided with induction heating. The dependence of the mechanical responses upon the prescribed temperatures and strain rates is presented and analysed.

\section{Parent material and specimen preparation}

The raw material was provided in form of plasma sprayed rectangular plates detached from any substrate. Cylindrical compression specimens of length $5 \mathrm{~mm}$ and diameter equal to $6 \mathrm{~mm}$ were extracted from the plates. Tensile dogbone specimens had gauge length equal to 8 $\mathrm{mm}$ and diameter of $3 \mathrm{~mm}$. All specimens were speckled using high temperature resistant coatings in order to allow for digital image correlation analysis.

\section{Experimental methods}

\subsection{Quasi-static tests}

Compression and tensile quasi static tests on METCO 601 abradable seal coating were conducted by means of a screw-driven universal testing machine used in conjunction with an environmental chamber equipped with resistive heating elements. The experiments were conducted at a nominal imposed strain rate of $1 \cdot 10^{-3} \mathrm{~s}^{-1}$ and at temperatures of $298 \mathrm{~K}, 373 \mathrm{~K}$ and $573 \mathrm{~K}$ in order to determine the sensitivity of the mechanical response of the material to temperature. The applied load was recorded by a resistive load cell while a non-contact laser extensometer was employed to record the nominal strains. All experiments were also recorded by a digital camera equipped with telecentric lenses in order to monitor the macroscopic strain field and the mechanisms of deformation and failure (Fig. 1). The temperature during the experiments was regulated automatically via a Eurotherm controller. Once the desired temperature was reached, it was maintained for approximately 20 minutes prior to testing in order to ensure a uniform temperature distribution within the specimen. The flat metal platens used to load the specimens during all compression tests were lubricated using a thin layer of high temperature grease in order to minimise friction.

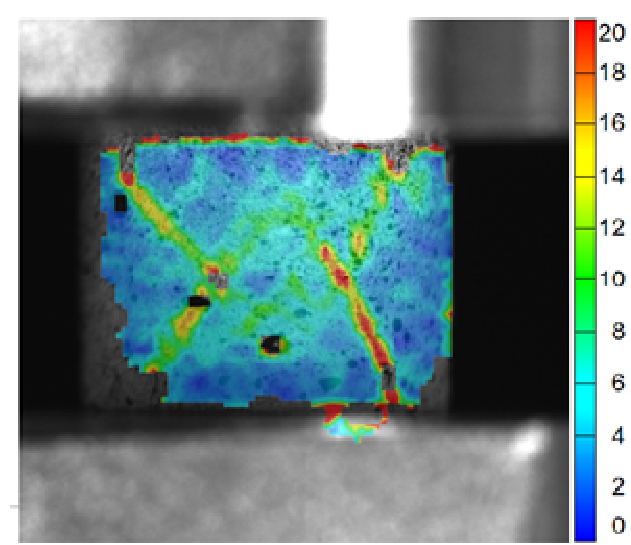

Fig. 1. Sulzer METCO 601. Distribution of equivalent Mises strain during incipient failure in compression.

\subsection{High-strain rate tests}

The compressive mechanical response of METCO 601 seal coating at strain rates representative of dynamic loading conditions (approximately $100 \mathrm{~s}^{-1}$ ) was assessed by conducting a series of laboratory experiments on the Split Hopkinson Pressure Bar apparatus. In this apparatus, an impactor is accelerated by a compressed air system and hits the input bars, generating a stress pulse of magnitude and duration determined by the impact velocity and by the geometrical and material properties of striker and input bar. The stress pulse travels along the input bar and, when reaching the specimen, is partially transmitted through the output bar and partially reflected back along the input bar. The strain rate, strain and stress histories in the specimen can determined from the recorded strain gauge signals via one dimensional wave analysis. Strain histories in the specimens were also measured via analysis of highspeed footage recorded employing an ultra-high-speed camera (Specialised Imaging -Kirana), which was typically operated at $2 \cdot 10^{5} \mathrm{fps}$.

The measurement of the dynamic response of abradable coatings at different temperatures presents some challenges. Firstly, abradable coatings are characterised by a low stress wave propagation speed and, therefore, require a relatively long time for the achievement of dynamic equilibrium conditions. This difficulty was overcome employing $1 \mathrm{~mm}$ thick neoprene pulse shapers and a SPHB comprised of bars and striker of considerable length. Specifically, the device used comprised of a striker and instrumented input and output bars of length and diameter equal to $2.7 \mathrm{~m}$ and $16 \mathrm{~mm}$ respectively. The noticeable length of the projectile inevitably leads to the superimposition of incident and reflected elastic stress waves into the input bar at the gauge location. A post-processing procedure, based on the method of characteristics and on D'Alambert's solution of wave equations, was used to calculate the magnitude of forward and backward travelling waves as functions of position and time. Detail on the procedure can be found in [8] where a similar setup was employed to measure the high strain rate compressive response of silica and volcanic sands [9]. 
Furthermore, the specimens were heated up to $373 \mathrm{~K}$ and $573 \mathrm{~K}$ using a feedback controlled induction heating system. In order to guarantee a uniform heating, the specimens were heated imposing a temperature rate of approximately $5{ }^{\circ} \mathrm{C} / \mathrm{s}$, monitored by means of type $\mathrm{K}$ thermocouples. Because of the composition of METCO 601 it was not possible to spot weld the thermocouples to the specimens, as it is usually done on metallic specimens. The contact of the thermocouples to the material was ensured bending the thermocouples wires into the shape of a small hook and preloading the thermocouples junction onto the specimen surface. In order to protect the bars from overheating during temperature conditioning, impedance matched tungsten carbide inserts were interposed between the specimen and the bars.

\section{Experimental results}

This section presents the stress-strain relationships measured during experiments on METCO 601 in order to assess the temperature and strain rate sensitivity of the material. Experiments conducted in each temperature and strain rate conditions were repeated a number of times in order to evaluate the variability on the material response. The measured stress is normalised with respect to the bond strength of the material [7], defined as the degree of adhesion of a coating to the substrate or the cohesion strength of the coating in a tension normal to the surface [10-11].

Representative stress strain curves obtained during quasi static compression experiments conducted at room temperature and at $373 \mathrm{~K}$ and $573 \mathrm{~K}$ are presented in Fig. 2. It is evident that, regardless the testing temperature, the material exhibits a higher strength compared to its nominal bond strength when tested in compression, as expected given the nature of the material, a blend of silicon-aluminium and polyester powders. In particular, at room temperature the ultimate strength of METCO 601 ranges between 7 and 7.94 times the bond strength and its strain to failure is included between $3 \%$ and 4.6 $\%$. It is noted that at room temperature the material fails in a brittle manner, showing evident shear bands prior to collapse (Fig. 1) and its capability to supports load drops immediately after failure, with the specimens breaking before crumbling under the compressive load. The curves obtained at $373 \mathrm{~K}$ show a visible softening of the material with ultimate stresses dropping to values included between 5.45 times and 5.72 times the bond strength with strains to failure between $6.7 \%$ and $8 \%$. It was noted that, while the failure mode was similar to the failure mode observed at room temperature the material was still able to support some of the compressive load after failure. Finally, the response measured at $573 \mathrm{~K}$ was characterised by an evident softening, with maximum stresses varying between 2.72 times and 3.52 times the bond strength and failure strains between $4.6 \%$ and $10 \%$.

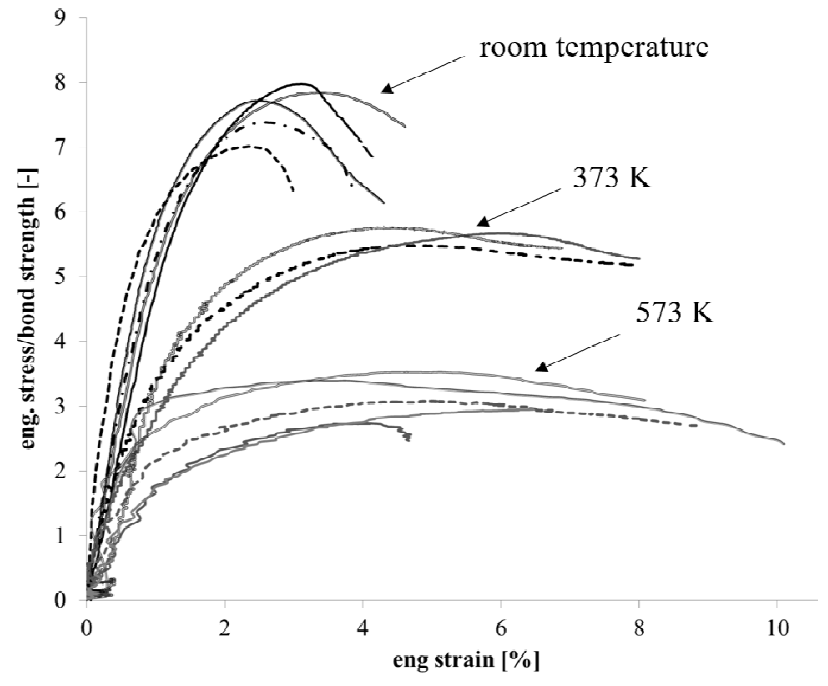

Fig. 2. Compressive quasi static mechanical response of METCO 601 abradable coating at different temperatures.

The mechanical behaviour of METCO 601 during quasi-static tensile experiments is summarised in Table 1. It is evident that the material fails in a brittle manner in tension and that it is characterised by a pronounced tension/compression asymmetry.

The validity of the dynamic experiments was verified by checking that the dynamic force equilibrium conditions were achieved during the experiments conducted on the SHPB apparatus. Fig. 3 presents time histories of the forces measured from the input and output bars for representative experiments conducted at the three temperatures of interest $(298 \mathrm{~K}, 373 \mathrm{~K}$ and 573 $\mathrm{K})$. The corresponding strain rate time histories are presented on the same charts. Dynamic equilibrium conditions were readily achieved during dynamic experiments conducted at room temperature and at 373 $\mathrm{K}$, with measured strain rates in the order of $100 \mathrm{~s}^{-1}$ (Fig. 3.a and Fig. 3.b). Validity conditions were also achieved during experiments conducted at $573 \mathrm{~K}$. However, due to the noticeable softening of the material and to pulse shaping technique adopted the measured strain rate was not constant (Fig. 3.c).

Table 1. Mechanical behaviour of METCO 601.

\begin{tabular}{|c|c|c|}
\hline temperature [K] & $\begin{array}{c}\text { peak stress / bond } \\
\text { strength [-] }\end{array}$ & $\begin{array}{c}\text { failure engineering } \\
\text { strain [\%] }\end{array}$ \\
\hline 298 & 1.80 & 0.30 \\
\hline 373 & 1.75 & 0.60 \\
\hline 573 & 0.99 & 0.75 \\
\hline
\end{tabular}


a)

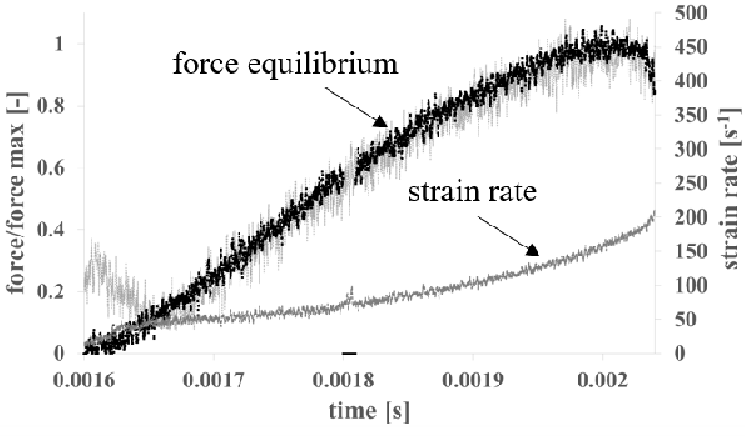

b)

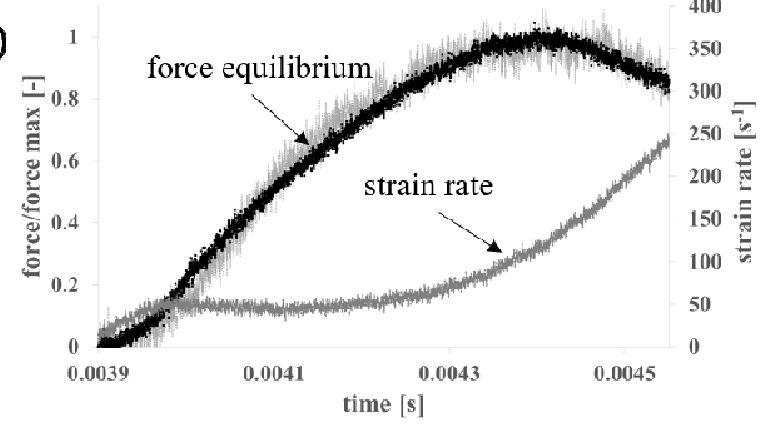

c)

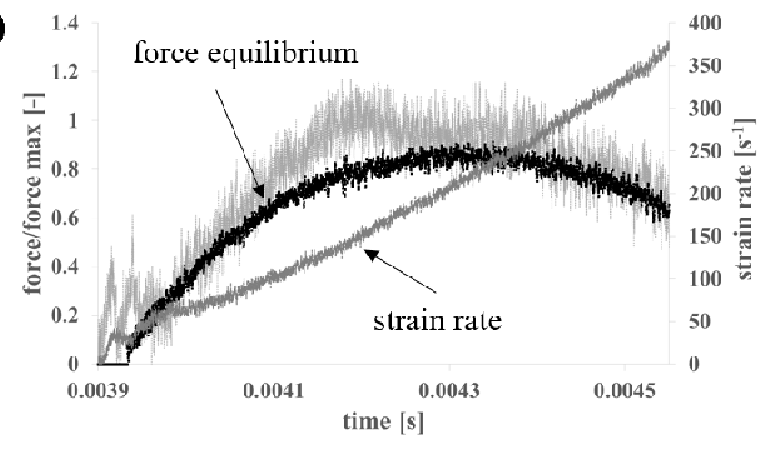

Fig. 3. Dynamic force equilibrium during SHPB experiments on METCO 601: a) room temperature, b) $373 \mathrm{~K}$, c) $573 \mathrm{~K}$.

The compressive responses of METCO 601 during quasi static and dynamic experiments are compared in Fig. 4. The material exhibits noticeable rate dependence at room temperature (Fig. 4.a) with the peak stresses measured during dynamic experiments approximately 25 $\%$ higher than the values obtained at quasi static rates of strain. Strains to failure were similar in quasi static and dynamic loading conditions. Experiments conducted at $373 \mathrm{~K}$ present even more pronounced rate dependence. In dynamic loading conditions the material shows a stiffer response and peak stresses up to $65 \%$ higher than in quasi static regime (Fig. 4.b). The material shows only moderate rate dependence at $573 \mathrm{~K}$ (Fig. 4.c).

\section{Conclusions}

An experimental campaign was undertaken in order to characterise the rate dependence of METCO 601 abradable coating at three different operating temperatures and motivate the development of theoretical models for this material. The main conclusions of this study are:

- The material exhibits noticeable rate dependence at room temperature and at $373 \mathrm{~K}$.
- The rate dependence of the compressive mechanical response of the coating is moderate at $573 \mathrm{~K}$.

-The material is characterised by a pronounced asymmetry of the mechanical behaviour in tension and compression.

a)

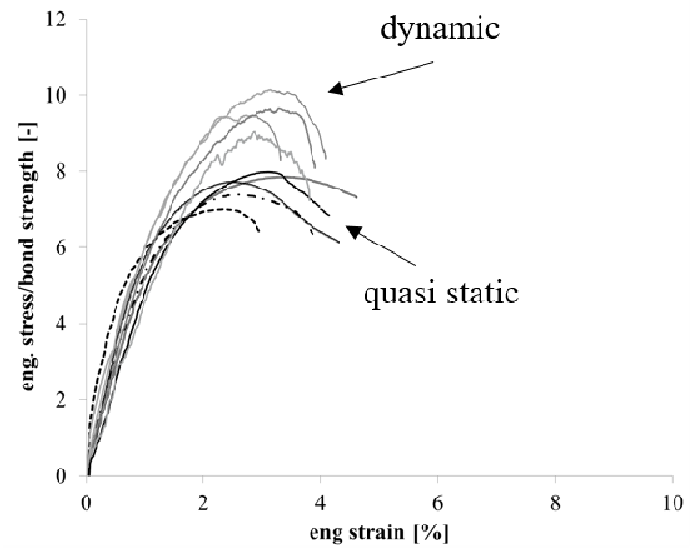

b)

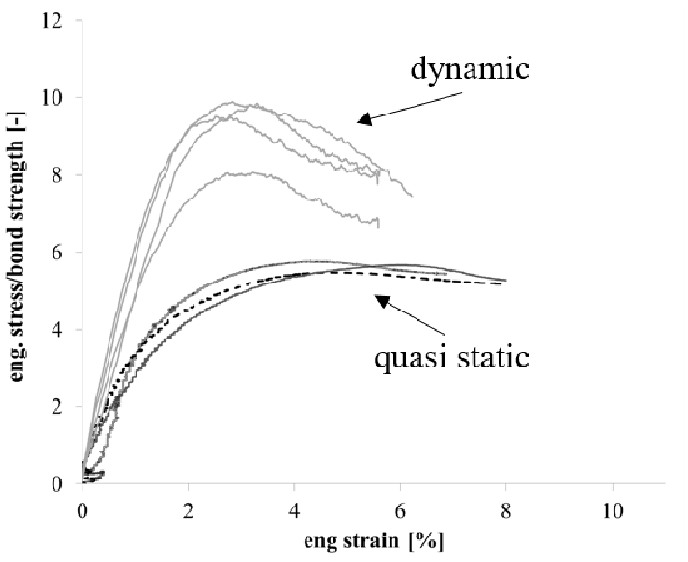

c)

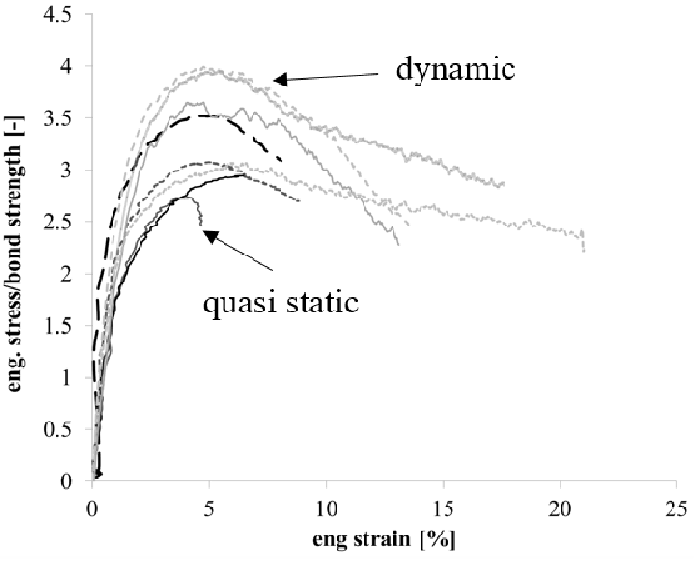

Fig. 4. Rate dependent compressive response of METCO 601. Quasi static (black) and dynamic (grey) mechanical response at a) room temperature, b) $373 \mathrm{~K}$, and c) $573 \mathrm{~K}$.

The authors would like to thank Mr. Stuart Carter and Mr. Jeffrey Fullerton for their assistance with fixture preparation and experimental setup and Mrs. Karen Bamford for helping everyone stay focused. 


\section{References}

1. R. Rajendran, Engineering Failure Analysis, 26 355369 (2012)

2. E. Lugscheider, J. Zwick, M. Hertter, D. Sporer, ITSC 2005: International Thermal Spray Conference 2005, Basel, Switzerland (2005)

3. M. Yi, J. He, B. Huang, H. Zhou, Wear, 231 47-53 (1999)

4. X. Ma, A. Matthews, Surface and Coatings Technology, 202 1214-1220 (2007)

5. Y. Maozhong, H. Baiyun, H. Jiawen, Wear, 252 915 (2002)

6. X. Ma, A. Matthews, Wear, 267 (2009) 1501-1510.

7. SulzerMetco ${ }^{\circledR}$, Metco ${ }^{\circledR}$ 601NS AluminumPolyester Powder. Technical Bulletin \#10-141 (2000)
8. F. De Cola, A. Pellegrino, C. Glößner, D. Penumadu, N. Petrinic, Experimental Mechanics, 58 223-242 (2018)

9. A. Pellegrino, F. De Cola, K. Dragnevski, N. Petrinic, Journal of Dynamic Behavior of Materials, 2 438-451 (2016)

10. ASTM C633-01 (2008). Standard test method for adhesion or cohesion strength of thermal spray coatings, A. International (2008)

11. W. Han, E.F. Rybicki, J.R. Shadley, Journal of Thermal Spray Technology, 2 145-150 (1993) 
